\title{
Proust ou la lecture comme expérience du Temps
}

\section{Luc Fraisse}

\section{(2) OpenEdition \\ Journals}

\section{Édition électronique}

URL : https://journals.openedition.org/studifrancesi/20607

DOI : $10.4000 /$ studifrancesi.20607

ISSN : 2427-5856

\section{Éditeur}

Rosenberg \& Sellier

\section{Édition imprimée}

Date de publication : 1 décembre 2019

Pagination : 485-495

ISSN : 0039-2944

\section{Référence électronique}

Luc Fraisse, "Proust ou la lecture comme expérience du Temps », Studi Francesi [En ligne], 189 (LXIII |

III) | 2019, mis en ligne le 01 décembre 2020, consulté le 12 novembre 2021. URL : http://

journals.openedition.org/studifrancesi/20607 ; DOI : https://doi.org/10.4000/studifrancesi.20607

\section{(c) $(1)(9)$}

Studi Francesi è distribuita con Licenza Creative Commons Attribuzione - Non commerciale - Non opere derivate 4.0 Internazionale. 


\title{
Proust ou la lecture comme expérience du Temps
}

\begin{abstract}
It is striking to note how in Proust's cycle of novels, the process of reading does not project the reader into the book that is being read, but leads rather to an extremely varied study of the act of reading itself, and principally of its duration. The very act of reading which is featured alongside the actual reading of a book, or indeed of a piece of music or a painting, can be broken down into several concurrent time-spans, which reflect the duration of real time as closely as possible. On a larger scale, the act of reading inaugurates an experience of time which can embrace and thus bring into contact the various chapters of the reader's life, if a book is re-read after a long interval of time (and this separates Proust's conception of time from that of Bergson). This duration is in its turn the measure of a work's posterity, which rests on a continuity of successive reading-lives. Hence, readers only exist for Proust "in Time", within a temporal dimension, that is in the form postulated a priori by Kant, of man's perception of time.
\end{abstract}

À la fin du Temps retrouvé, le héros de la Recherche, à présent décidé à entreprendre une grande œuvre, comprend que le sujet qui lui manquait autrefois à Combray - «tâchant de trouver un sujet où je pusse faire tenir une signification philosophique infinie» ${ }^{1}-$, sera de rendre sensible la substance invisible du Temps ${ }^{2}$. L'œuvre à écrire prendra elle-même cette forme du Temps, c'est-à-dire que «ce serait un livre aussi long que Les Mille et une Nuits» ${ }^{3}$, donc essentiellement «long à écrire» ${ }^{4}$ - et donc encore long à lire. Du reste, le temps de l'écriture et celui de la lecture se rejoignent pour se confondre, si l'on remarque avec Pascal Alain Ifri que le cycle romanesque tout entier pourrait bien avoir pour sujet général un apprentissage de la lecture 5 Lecture au sens large sans doute, incluant la lecture du monde, cet univers de signes à déchiffrer qu'a isolés chez Proust Gille Deleuze dans un essai célèbre 6 .

Mais si l'on restreint la notion de lecture à celle qui s'applique à un livre, on remarquera que sous la plume de Proust, l'acte de la lecture porte en lui-même l'empreinte du Temps - et concourt à le rendre visible -, et ce semble-t-il de mille manières diverses. C'est donc de la lecture considérée comme une expérience du Temps qu'il sera question ici. Dans cette grande variété de cas et de situations, il semble possible de distinguer deux domaines complémentaires mais différents: l'expérience temporelle de la lecture, c'est-à-dire l'expérience du temps qui passe au moment même où on lit; et au-delà ou en deçà de ce moment, les liens unissant l'activité de la lecture à une expérience du Temps sur une beaucoup plus grande échelle.

(1) À la recherche du temps perdu, édition réalisée sous la direction de Jean-Yves Tadié, Paris, Gallimard,

1987-1989, «Bibliothèque de la Pléiade», 4 voll., t. I, p. 170.

(2) Ibidem, t. IV, p. 622.

(3) Ibidem, p. 621.

(4) Ibidem, p. 620.

(5) Voir P.A. Ifri, Proust et son narrataire dans "À la recherche du temps perdu”, Genève, Droz, 1983: «le thème central du roman, celui de la lecture» (p. 243).

(6) Marcel Proust et les signes, Paris, Puf, 1964. 
Une originalité assez spécifique au roman de Proust est de détailler le temps de la lecture considéré au moment où elle s'accomplit. C'est-à-dire qu'ici, cette activité d'ordinaire évoquée sous le seul angle de la plongée dans ce qui est lu, est décrite de façon réflexive par rapport à la conscience de celui qui est en train de lire - de même que, dans les scènes de concert ménagées de loin en loin dans la Recherche, une partie essentielle de ces séquences est pour évoquer, non la musique ou les musiciens, mais l'auditoire.

C'est ainsi que les lectures du héros enfant à Combray permettent de distinguer, chez celui qui est en train de lire, deux durées simultanées, qui seraient le temps de la lecture et le temps vécu:

à chaque heure il me semblait que c'était quelques instants seulement auparavant que la précédente avait sonné; la plus récente venait s'inscrire tout près de l'autre dans le ciel et je ne pouvais croire que soixante minutes eussent tenu dans ce petit arc bleu qui était compris entre leurs deux marques d'or. Quelquefois même cette heure prématurée sonnait deux coups de plus que la dernière; il y en avait donc une que je n'avais pas entendue, quelque chose qui avait eu lieu n'avait pas eu lieu pour $\mathrm{moi}^{\top}$.

Et au sein de cette expérience même il serait loisible de distinguer encore plusieurs durées, ou plusieurs dimensions de la durée, dans la mesure où il existe plusieurs vitesses de lecture, comme on le voit quand un roman tient sous son entière dépendance, «tandis que nous tournons fiévreusement les pages du livre, la rapidité de notre respiration et l'intensité de notre regard» ${ }^{8}$.

Il faudrait se souvenir ici que le rival de Proust dans son époque concernant la théorie de la mémoire, son cousin Henri Bergson, recourt précisément au temps exact de la lecture pour distinguer, dans Matière et mémoire en 1896, le souvenir habitude et le souvenir image. Voici en effet l'exemple simple et célèbre que choisit le philosophe:

J'étudie une leçon, et pour l'apprendre par cœur je la lis d'abord en scandant chaque vers; je la répète ensuite un certain nombre de fois. À chaque nouvelle lecture un progrès s'accomplit; les mots se lient de mieux en mieux; ils finissent par s'organiser ensemble. A ce moment précis je sais ma leçon par cœur; on dit qu'elle est devenue souvenir, qu'elle s'est imprimée dans ma mémoire. I Je cherche maintenant comment la leçon a été apprise, et je me représente les phases par lesquelles j'ai passé tour à tour. Chacune des lectures successives me revient alors à l'esprit avec son individualité propre; je la revois avec les circonstances qui l'accompagnaient et qui l'encadrent encore; elle se distingue de celles qui précèdent et de celles qui suivent par la place même qu'elle a occupée dans le temps; bref, chacune de ces lectures repasse devant moi comme un événement déterminé de mon histoire. On dira encore que ces images sont des souvenirs, qu'elles se sont imprimées dans ma mémoire. On emploie les mêmes mots dans les deux cas. S'agit-il bien de la même chose?'

Proust, au moment où paraît Du côté de chez Swann en novembre 1913, dans une entrevue accordée à Élie-Joseph Bois pour Le Temps, insiste, avec raison autant que par stratégie, sur la différence entre les deux formes de mémoire distinguées par

(7) Recherche, t. I, pp. 86-87.

(8) Ibidem, p. 84.

(9) Matière et mémoire. Essai sur la relation du corps à l'esprit, Paris, Félix Alcan, 1896, «Bibliothèque de philosophie contemporaine», rééd. Paris, Puf, 1941, pp. 83-84. 
Bergson et les mémoires volontaire et involontaire distinguées à travers l'épisode de la madeleine ${ }^{10}$.

Une rencontre au sommet de la pensée française se déroule donc autour d'une expérience de la lecture, plus précisément du temps de la lecture, mais on verra plus loin qu'à ce stade se dégage l'originalité de l'usage que fait le romancier de cette expérience, par rapport au lien entre temps et lecture qu'établit en général la philosophie toute contemporaine.

À partir de là, le romancier de la Recherche multiplie les cas inattendus, mais ayant en commun de se fixer sur ces instants vécus au présent par le lecteur en train de lire. Qui pourrait ainsi penser que le mouvement de l'œil sur la page puisse constituer en soi une aventure spirituelle? C'est pourtant ce qui ressort de cet exemple, que prend le narrateur des Jeunes filles en fleurs pour prouver la différence, s'agissant de l'écrivain qu'admire entre tous le héros dans sa jeunesse, entre «le Bergotte» et le style «à la Bergotte», c'est-à-dire entre les véritables phrases de Bergotte et les phrases de ses imitateurs, différence éclairée par analogie avec Saint-Simon:

Un auteur de Mémoires d'aujourd'hui, voulant sans trop en avoir l'air, faire du Saint-Simon, pourra à la rigueur écrire la première ligne du portrait de Villars: «C'était un assez grand homme brun... avec une physionomie vive, ouverte, sortante», mais quel déterminisme pourra lui faire trouver la seconde ligne qui commence par: «et véritablement un peu folle»? ${ }^{11}$

Et en effet, on peut vérifier cet événement de lecture. Pour comprendre pleinement ces considérations, il faut se reporter à l'édition Chéruel $^{12}$, qui est celle que possède Proust, et observer le découpage matériel du passage, à cheval sur deux pages: «C'était un assez grand homme, brun, I bien fait, devenu gros en vieillissant, sans en être appe- II santi, avec une physionomie vive, ouverte, sortante, et I véritablement un peu folle». C'est-à-dire que la mise en page et en lignes, purement fortuite pourtant, de ce portrait, le romancier, qui en cela frôle la mauvaise foi, les met au service de l'originalité de Saint-Simon, le retour à la ligne de l'édition Chéruel détachant la formule la plus inattendue comme le ferait volontairement un poète par un rejet. Cette surprise de l'œil, due à une répartition typographique purement aléatoire, est reportée au compte de l'esprit d'invention du mémorialiste. C'est à la faveur de ce raisonnement discrètement abracadabrantesque que le temps de la lecture peut se peupler de découvertes et de coups de théâtre.

Il faut dire que souvent, dans la Recherche, le temps de la lecture restitué dans sa durée réelle est transporté dans l'examen ou l'inspection d'un tableau, l'œil se promenant de place en place sur la toile comme il suivrait, quoiqu'ici plus librement mais avec le même détail, les lignes d'un livre. Observons sous ce jour l'écrivain Bergotte devant la Vue de Delft de Vermeer: il s'est rendu à l'exposition

un critique ayant écrit que dans la Vue de Delft de Ver Meer (prêté par le musée de La Haye pour une exposition hollandaise), tableau qu'il adorait et croyait connaitre très bien, un

(10) Voir Essais et articles, publiés avec Contre Sainte-Beuve et Pastiches et Mélanges par P. Clarac et Y. Sandre, Paris, Gallimard, 1971, «Bibliothèque de la Pléiade», p. 558: «mon œuvre est dominée par la distinction entre la mémoire involontaire et la mémoire volontaire, distinction qui non seulement ne figure pas dans la philosophie de M. Bergson, mais est même contredite par elle».

(11) Recherche, t. I, p. 541.

(12) Mémoires du duc de Saint-Simon, édités pour la première fois d'après les manuscrits originaux, précédés d'une notice par Sainte-Beuve, par A. Chéruel, Paris, Louis Hachette, 20 voll., 1856-1858, rééd. 22 voll. avec la collaboration d'A. Régnier, 1873-1886, t. IV, pp. 28-29. Voir Saint-Simon, Mémoires, édition établie par Y. Coirault, Paris, Gallimard, 1983-1988, «Bibliothèque de la Pléiade», 8 voll., t. II, p. 252. 
petit pan de mur jaune (qu'il ne se rappelait pas) était si bien peint qu'il était, si on le regardait seul, comme une précieuse œuvre d'art chinoise, d'une beauté qui se suffirait à elle-même;

or, «grâce à l'article du critique, il remarqua pour la première fois des petits personnages en bleu, que le sable était rose, et enfin la précieuse matière du tout petit pan de mur jaune» ${ }^{13}$ qui constitue ici, mais cette fois dans l'espace du tableau, le coup de théâtre de pareille lecture.

Observons parallèlement, dans La Fugitive, le héros à Venise devant une toile de Carpaccio:

Je voyais pour la première fois Le Patriarche di Grado exorcisant un possédé. Je regardais l'admirable ciel incarnat et violet sur lequel se détachent ces hautes cheminées incrustées, dont la forme évasée et le rouge épanouissement de tulipes fait penser à tant de Venises de Whistler. Puis mes yeux allaient du vieux Rialto en bois à ce Ponte Vecchio du Xv siècle aux palais de marbre ornés de chapiteaux dorés, revenaient au canal où les barques sont menées par des adolescents en vestes roses, en toques surmontées d'aigrettes [...]. Enfin, avant de quitter le tableau mes yeux revinrent à la rive où fourmillent les scènes de la vie vénitienne de l'époque. Je regardais le barbier essuyer son rasoir, le nègre portant son tonneau, les conversations des musulmans, des nobles seigneurs vénitiens en larges brocarts, en damas, en toque de velours cerise, quand tout à coup je sentis au cœur comme une légère morsure ${ }^{14}$.

Le coup de théâtre de cette lecture aléatoire est ici que la toile renferme le modèle de la robe de Fortuny qu'avait offerte le héros à Albertine aujourd'hui défunte.

Cette errance méthodique de l'œil sur la toile, qui rapproche son observation d'une lecture à la recherche de son explication, se nourrit de la description de tableaux par les frères Goncourt, que Robert Ricatte a appelée la description ambulatoire $^{15}$. C'est cette promenade organisée et analytique de place en place sur la toile qui rapproche le regard de l'esthète de l'activité du lecteur elle-même observée dans la durée de sa procédure.

Il est un cas où la lecture du tableau vient exactement se superposer au temps de la lecture - non alors celle du héros, mais de nous lecteurs. C'est le moment, décisif, où, lors de sa deuxième visite à Odette (dans le but de lui montrer une gravure), Swann s'avise de la ressemblance de la jeune femme avec la Zéphora de Botticelli. L'analogie surgit ainsi:

Debout à côté de lui, laissant couler le long de ses joues ses cheveux qu'elle avait dénoués, fléchissant une jambe dans une attitude légèrement dansante pour pouvoir se pencher sans fatigue vers la gravure qu'elle regardait, en inclinant la tête, de ses grands yeux, si fatigués et maussades quand elle ne s'animait pas, elle frappa Swann par sa ressemblance avec cette figure de Zéphora, la fille de Jéthro, qu'on voit dans une fresque de la chapelle Sixtine ${ }^{16}$.

Le romancier sait que son personnage fictif pourra dès lors être confronté à la figure réellement peinte par Botticelli, reproduite d'ailleurs au fronton d'un volume (le t. XXIII) des œuvres complètes de Ruskin, dans l'édition anglaise qu'il possédait ${ }^{17}$. Le

(13) Recherche, t. III, p. 692.

(14) Ibidem, t. IV, pp. 225-226.

(15) Voir R. Ricatte, La Création romanesque chez les Goncourt, Paris, Armand Colin, 1953, notamment pp. 280 et 415.

(16) Recherche, t. I, p. 219

(17) Voir J. Bastianelli, Dictionnaire Proust-Ruskin, Paris, Classiques Garnier, 2017, «Bibliothèque proustienne», p. 121. 
procédé d'écriture consiste en conséquence à partir de ce point d'arrivée, et à rétrograder dans la description d'Odette selon un acheminement qui se terminera par une coïncidence parfaite avec la figure peinte. Aussi la phrase descriptive s'encombret-elle d'un grand nombre de notations qui œuvrent à retarder ce résultat, à le faire advenir lentement. Et ainsi, la superposition de la phrase et de la peinture se forme dans l'étirement du temps de la lecture au moment même où elle s'accomplit.

Deux autres situations originales mettent en scène la lecture considérée, non comme un concept, mais comme une activité installée dans la durée. Dans Le Côté de Guermantes, le héros, habitué depuis son enfance aux images et au style de Bergotte, en est à découvrir un «nouvel écrivain». La mise en scène du temps nécessaire au parcours de la phrase (moins ici de l'œil que par la pensée), pour pénétrer le sens, sert maintenant à donner à apercevoir dans son épaisseur l'effort de lecture:

je sentais que ce n'était pas la phrase qui était mal faite, mais moi pas assez fort et agile pour aller jusqu'au bout. Je reprenais mon élan, m'aidais des pieds et des mains pour arriver à l'endroit d'où je verrais les rapports nouveaux entre les choses. Chaque fois, parvenu à peu près à la moitié de la phrase, je retombais, comme plus tard au régiment dans l'exercice appelé portique $^{18}$.

Ici, l'insaisissable activité de la lecture a besoin d'une imagerie physique pour parvenir à être perçue, en un temps qui n'est pas autrement mesurable que par son arrêt à mi-course. Ce sont l'effort et la sensation de résistance qui rendent ici sensibles, non seulement la substance du temps, mais celle du temps de la lecture en train de s'accomplir.

Parce que la lecture est, sous la plume de Proust, un moment d'effort, là où généralement on la représente en se projetant dans ce qui est lu, comme si la lecture en elle-même n'existait pas, ce temps se redouble et se multiplie en raison de la nécessité, pour approfondir l'œuvre lue, bien évidemment des relectures. Et ici Proust, on va le voir, se fait bergsonien. Parce que le temps de la lecture est celui de l'assimilation de l'œuvre, on peut dire à plusieurs titres, avec le narrateur d'À l'ombre des jeunes filles en fleurs, de la lecture qu'il en est d'elle «comme tout ce qui se réalise dans le temps» ${ }^{19}$. Pour le donner à comprendre, le romancier transpose à nouveau le processus de la lecture, mais cette fois dans l'appréhension d'une ligne mélodique, au moment où le héros, introduit chez les Swann, voit et entend Odette jouer au piano la sonate de Vinteuil, dans cet épisode qu'on appelle volontiers la «scène de passation» de la sonate, par Swann et Odette au héros qui en prolongera bien plus tard l'enseignement à l'audition du septuor dans La Prisonnière.

Tandis que le héros entend pour la première fois cette sonate, au contraire si familière à Swann et Odette qui la lui commentent, le narrateur prend le relais pour philosopher sur les différentes mesures de temps qu'il faudrait adopter afin de cerner les «relectures» successives de l'œuvre à appréhender, une œuvre ici musicale qui rend compte aussi bien de la lecture et de la relecture d'une œuvre littéraire, telles ces phrases du «nouvel écrivain» péniblement parcourues, on l'a vu, par le héros dans le volume suivant. Dès ici à propos de Vinteuil, cette même résistance de l'œuvre à la première «lecture» est ainsi analysée: «Mais souvent on n'entend rien, si c'est une musique un peu compliquée qu'on écoute pour la première fois»; et cependant, ajoute le narrateur, «si l'on n'avait vraiment, comme on l'a cru, rien distingué à la pre-

(18) Recherche, t. II, pp. 622-623.

(19) Ibidem, t. I, p. 521. 
mière audition, la deuxième, la troisième seraient autant de premières, et il n'y aurait pas de raison pour qu'on comprît quelque chose de plus à la dixième» ${ }^{20}$.

C'est cet aspect de la réflexion sur les différents temps des lectures successives qui est éminemment bergsonien, une réflexion en forme d'objection à la quantification numérique (ce que Bergson appellerait le temps d'horloge, divisant les moments en segments égaux), au profit d'une durée vécue à vitesses variables. Par quoi le temps d'une relecture ne reproduit pas celui de la première lecture, et ce phénomène constaté donne accès à l'essence qualitative de la durée - ce que Bergson appellerait maintenant la pure durée. Comme le temps de relecture selon Proust n'est pas le temps de la première lecture qui serait reproduit à l'identique, l'alternative pour Bergson n'est pas le choix entre deux possibilités équidistantes, puisqu'il faut tenir compte (ce que l'on oublie de faire) que l'itinéraire s'étoffe entre l'examen de la première position et celui de la seconde, si bien que l'examen de la seconde position s'est obligatoirement enrichi de l'examen de la première.

La vérité, souligne l'auteur de l'Essai sur les données immédiates de la conscience, est que le moi, par cela seul qu'il a éprouvé le premier sentiment, a déjà quelque peu changé quand le second survient: à tous les moments de délibération, le moi se modifie et modifie aussi, par conséquent, les deux sentiments qui l'agitent. Ainsi se forme une série dynamique d'états qui se pénètrent, se renforcent les uns les autres ${ }^{21}$.

Lire et relire, écouter et réécouter, donnent ainsi accès, par l'entremise de l'art, à une approche affinée des nuances de la durée vécue.

Il est une expérience originale, ménagée par le narrateur de La Fugitive, donnant à apercevoir dans le détail ce temps spécifique de la relecture - originale parce qu'il s'agit en l'occurrence d'une relecture de soi-même. On sait que paraît enfin, dans Le Figaro, le poème en prose sur les clochers de Martinville, composé en hâte autrefois à Combray, apparemment bien oublié depuis. Plusieurs pages de cet avant-dernier volume du cycle romanesque décrivent à loisir cette expérience, pour un auteur, de relire un texte ancien de soi-même. Sur un ton humoristique d'auto-dérision, le narrateur trouve à compléter ses réponses à la question de savoir quelle durée traverse le lecteur analysé en train de lire.

La situation particulière, du lecteur qui en fait se relit, fait apparaître que le temps de la lecture se dédouble en direction d'une autre dimension, qui est (n'en déplaise cette fois à Bergson) sa projection dans l'espace, c'est-à-dire dans un monde d'images. Non plus les images des paysages décrits dans le texte lu, mais, étant donné cette situation particulière, la représentation, simultanée à la lecture, des autres lecteurs devant lire ce même texte. Il en résulte aussitôt un temps démultiplié par le groupe de lecteurs que l'auteur se représente et à qui il distribue, en quelque sorte, on va le voir, ses phrases:

Si M. de Guermantes ne comprenait pas telle phrase que Bloch aimerait, en revanche il pourrait s'amuser de telle réflexion que Bloch dédaignerait. Ainsi pour chaque partie que le lecteur précédent semblait délaisser, un nouvel amateur se présentant, l'ensemble de l'article se trouvait élevé aux nues par une foule ${ }^{22}$.

(20) Ibidem, p. 520.

(21) H. Bergson, Essai sur les données immédiates de la conscience, Paris, Félix Alcan, 1889, rééd. Paris, Puf, 1976, p. 129.

(22) Recherche, t. IV, p. 149. 
C'est cette variété des expériences temporelles simultanées qui ouvre, avec une pointe d'humour non dissimulée (une tournure syntaxique désuète, classique, pascalienne - n'y ayant rien -, aboutissant à une citation de Musset), à l'expérience de la relecture:

Aussi, à peine eus-je fini cette lecture réconfortante, que moi qui n'avais pas eu le courage de relire mon manuscrit, je souhaitai de la recommencer immédiatement, n'y ayant rien comme un vieil article de soi dont on puisse dire que "quand on l'a lu on peut le relire"23.

L'expérience de la relecture à distance se complète ici par celle de la relecture immédiate. L'une et l'autre sont en quelque sorte manquées par le parasitage du rapport de soi à soi. La durée vécue personnelle au sujet qui lit perturberait-elle la durée actuelle de la lecture?

Non nécessairement, car la même expérience se reproduit, mais expurgée de ce lien encombrant à soi-même, presque à l'issue du Temps retrouvé, quand le héros à présent vieilli aperçoit, dans la bibliothèque du prince de Guermantes, un exemplaire de François le Champi identique à celui que sa mère lui avait lu, il y a bien longtemps, une nuit à Combray. Le narrateur philosophe ainsi sur cet épisode en quelque sorte de mémoire involontaire, mais volontairement reproductible, que permet la redécouverte d'une lecture ancienne:

si je reprends dans la bibliothèque François le Champi, immédiatement en moi un enfant se lève qui prend ma place, qui seul a le droit de lire ce titre: François le Champi, et qui le lit comme il le lut alors, avec la même impression du temps qu'il faisait dans le jardin, les mêmes rêves qu'il formait alors sur les pays et sur la vie, la même angoisse du lendemain ${ }^{24}$;

d'où résulte cette réplique sous-entendue à la bibliophilie des frères Goncourt:

La première édition d'un ouvrage m'eût été plus précieuse que les autres, mais j'aurais entendu par elle l'édition où je le lus pour la première fois. Je chercherais les éditions originales, je veux dire celles où j'eus de ce livre une impression originale ${ }^{25}$.

Une édition originale en ce sens renouvelé produit, remarquons-le, dans la durée vécue du lecteur, un souvenir image selon Bergson. Mais là apparaît cependant l'originalité de Proust théoricien de la mémoire, car chez Bergson et chez tous les philosophes contemporains de la Recherche, le souvenir ressuscité fût-ce spontanément reste très récent. C'est pourquoi le narrateur du Temps retrouvé ira se chercher des précurseurs, non dans l'histoire de la philosophie, mais chez certains de ses maitres en littérature, tels Chateaubriand, Nerval et Baudelaire ${ }^{26}$. De fait, dans les lectures et relectures qui permettent à Bergson de distinguer le souvenir habitude du souvenir image, quelques heures d'écart suffisent pour englober l'ensemble du phénomène. Les deux lectures de François le Champi au contraire font se rejoindre, par-delà les années, les deux extrêmes de la vie du héros proustien.

(24) Ibidem, p. 464

(25) Ibidem, p. 465.

(26) Ibidem, pp. 498-499. 
Car, par un élargissement soudain, si une spécificité de Proust romancier semble bien la description de l'acte de lire dans sa durée réelle, laquelle en un court instant trouve à renfermer un monde, le temps de la lecture prend sa véritable dimension à l'échelle de toute une vie. Le théoricien part de la conception classique de la lecture de romans comme concentration de la vie: ainsi, à Combray, «ces après-midi-là étaient plus remplis d'événements que ne l'est souvent toute une vie» ${ }^{27}$, parce qu'un roman

déchaîne en nous pendant une heure tous les bonheurs et tous les malheurs possibles dont nous mettrions dans la vie des années à connaître quelques-uns, et dont les plus intenses ne nous seraient jamais révélés parce que la lenteur avec laquelle ils se produisent nous en ôte la perception ${ }^{28}$;

c'est-à-dire que la concentration de la vie dans un roman ajoute, à ses étapes successives, «la sensation même du changement ${ }^{29}$. On pourrait reconnaître ici la fameuse remarque de Diderot dans son Éloge de Richardson: «J'avais parcouru dans l'intervalle de quelques heures un grand nombre de situations, que la vie la plus longue offre à peine dans toute sa durée ${ }^{30}$. Mais on voit combien cette expérience de la concentration du temps par la lecture des romans s'est trouvée depuis approfondie par un siècle et demi de science psychologique.

À cette échelle, une lecture est conçue par Proust comme une étape de vie. À Combray, il y a l'automne de la mort de la tante Léonie, coloré par la lecture d'Augustin Thierry ${ }^{31}$; et Proust explique ainsi à un ami la lecture de François le Champi: «Ne croyez pas que j'aime George Sand. Ce n'est pas un morceau de critique. C'est comme cela à cette date-là. Le reste du livre corrigera» ${ }^{32}$. Aussi le texte «Journées de lecture» composant la préface à Sésame et les lys (1906) définit-il le temps vécu, mais à long terme, de la lecture comme une fin qui est un commencement:

pour l'auteur ils [les livres] pourraient s'appeler "Conclusions" et pour le lecteur "Incitations". Nous sentons très bien que notre sagesse commence où celle de l'auteur finit et nous voudrions qu'il nous donnât des réponses, quand tout ce qu'il peut faire est de nous donner des désirs ${ }^{33}$.

La lecture entretient un rapport inchoatif au temps, au point que l'on rencontre, dans $\grave{A}$ l'ombre des jeunes filles en fleurs, le cas extrême d'une lecture qui est projection dans l'avenir, quand le héros entend l'actrice la Berma jouer une pièce toute contemporaine et repère chaque tirade «qui serait un jour fameuse», car alors «à défaut de la célébrité qu'elle n'avait pas pu avoir dans le passé, j'ajoutais celle qu'elle aurait dans l'avenir, par un effort d'esprit inverse de celui qui consiste à se représenter des chefs-d'œuvre au temps de leur grêle apparition» ${ }^{34}$.

(27) Ibidem, t. I, p. 83.

(28) Ibidem, p. 84

(29) Ibidem, p. 85.

(30) L'Éloge de Richardson a paru en janvier 1762 dans le Journal étranger. Voir Denis Diderot, Euvres, édition établie et annotée par A. Billy, Paris, Gallimard, 1951, «Bibliothèque de la Pléiade», pp. 1059-1074, ici p. 1060.

(31) Recherche, t. III, p. 231; c'est une révélation rétrospective dans La Prisonnière: «à Combray tandis qu'avant de partir marcher du côté de Méséglise, je lisais Augustin Thierry».

(32) Correspondance de Marcel Proust, établie, annotée et préfacée par Ph. Kolb, Paris, Plon, 1970-1993, 21 voll., t. IX, p. 225

(33) Pastiches et Mélanges cit., p. 176.

(34) Recherche, t. II, p. 350. 
Aux dimensions d'une vie de lecture, celles de l'enfance dessinent en filigrane un véritable art poétique romanesque. Le préfacier de Ruskin déclarait en effet:

Il n'y a peut-être pas de jours de notre enfance que nous ayons si pleinement vécus que ceux que nous avons cru laisser sans les vivre, ceux que nous avons passés avec un livre préféré. Tout ce qui, semblait-il, les remplissait pour les autres, et que nous écartions comme un obstacle vulgaire à un plaisir divin ${ }^{35}$.

C'est-à-dire que l'épaisseur de temps enrobant de telles lectures opère cette décantation qu'attend du roman cette génération qui s'éloigne à présent de l'école réaliste. Proust écrit parallèlement, dans une lettre à Robert Dreyfus, fin décembre 1913: «Pas une seule fois un de mes personnages ne ferme une fenêtre, ne se lave les mains, ne passe un pardessus, ne dit une formule de présentation. S'il y avait même quelque chose de nouveau dans ce livre, ce serait cela» ${ }^{36}$.

C'est encore le rapport de la lecture au temps qui a induit, esquissé cette conception renouvelée du roman.

À une échelle encore plus grande, le temps de la lecture en se déployant dessine, avec ses rebondissements, la vie des œuvres. Car le temps de l'assimilation des œuvres est une des formes du temps de la lecture, un temps simplement étalé sur une série de générations placées en continuité. La «scène de transmission» de la sonate de Vinteuil, de Swann et Odette au héros, élargit le temps individuel de la première lecture d'une œuvre au temps pour finir multiséculaire de sa postérité, cette seconde durée à long terme étant la conséquence esthétique de la première: «Et non seulement on ne retient pas tout de suite les œuvres vraiment rares, mais même au sein de chacune de ces œuvres-là, et cela m'arriva pour la Sonate de Vinteuil, ce sont les parties les moins précieuses qu'on perçoit d'abord $\aleph^{37}$. Voilà pour le temps individuel de la première lecture, qui conditionne déjà le déroulement, ultérieur, de la postérité:

Ce temps du reste qu'il faut à un individu - comme il me le fallut à moi à l'égard de cette Sonate - pour pénétrer une œuvre un peu profonde, n'est que le raccourci et comme le symbole des années, des siècles parfois, qui s'écoulent avant que le public puisse aimer un chef-d'œuvre vraiment nouveau» ${ }^{38}$;

si bien qu'il faudra attendre que les œuvres s'uniformisent en quelque sorte dans le passé artistique, «en une matière variée sans doute, mais somme toute homogène, où Hugo voisine avec Molière» ${ }^{39}$.

Encore la patine du temps n'homogénéisera-t-elle jamais définitivement les œuvres du passé, parce que la postérité ne cessera - ultime manifestation du temps dans le processus de la lecture - d'y dégager des sens nouveaux, ce que souligne Proust épistolier, à l'heure pourtant où est en train de triompher l'histoire littéraire de Gustave Lanson:

Sans doute il ne faut pas qu'elle [la critique] aille à l'encontre des tendances, de la ligne de croissance, d'un artiste. [...] Mais il nous est permis de faire goûter dans les tragédies de

(35) Pastiches et Mélanges cit., p. 160.

(36) Correspondance, t. II, p. 394.

(37) Recherche, t. I, p. 521.

(38) Ibidem, pp. 521-522.

(39) Ibidem, p. 523. 
Racine, dans ses Cantiques, dans les lettres de Mme de Sévigné, dans Boileau, des beautés qui s'y trouvent réellement et que le XVII siècle n'a guère aperçues ${ }^{40}$.

L'histoire littéraire postule, à la même époque, que les œuvres ont un sens, et que le sens de l'œuvre est celui que leur auteur a voulu lui donner et que son premier public était susceptible d'entendre. Or, pour un écrivain tel que Proust, le sens des œuvres ne cesse d'augmenter, parce que le temps en travaille la pâte comme le ferait un levain.

Au point que le temps de la lecture produit de bénéfiques brusqueries. Il est bon, suggère indirectement Proust, après avoir traversé la nappe de durée de la lecture continue, cursive, de rompre pareille continuité harmonieuse en désactualisant, par la simple citation, telle pièce de l'ensemble qui trouve un relief nouveau, pourraiton dire, projeté dans ce paysage asynchronique. Telle est la force découverte dans des passages isolés au moyen d'une citation, à propos des vers de Victor Hugo que cite la duchesse dans Le Côté de Guermantes:

en citant ainsi un vers isolé on décuple sa puissance attractive. Ceux qui étaient entrés ou rentrés dans ma mémoire, au cours de ce dîner, aimantaient à leur tour, appelaient à eux avec une telle force les pièces au milieu desquelles ils avaient l'habitude d'être enclavés, que mes mains électrisées ne purent pas résister plus de quarante-huit heures à la force qui les conduisait vers le volume où étaient reliés Les Orientales et Les Chants du crépuscule ${ }^{41}$.

Ces ruptures de chaîne chronologique constitueraient, à en croire l'écrivain et son narrateur, peut-être le seul moyen d'atteindre au plus profond l'originalité d'un auteur du passé - car, on va le voir, il faut du temps passé pour rendre possible la démonstration, bien connue, s'agissant du côté Dostoïevski des lettres de Mme de Sévigné: la marquise épistolière, comme le peintre impressionniste de la Recherche Elstir, présente «les choses, dans l'ordre de nos perceptions, au lieu de les expliquer d'abord par leur cause», comme ce paysage sous la lune: «je vois mille coquecigrues, des moines blancs et noirs, plusieurs religieuses grises et blanches, du linge jeté parci par-là, des hommes ensevelis tout droits contre des arbres $\rangle^{42}$. Il faut redistribuer entièrement les cartes de la chronologie pour apercevoir ces étranges anticipations. Ici, ce n'est pas seulement le lecteur de l'avenir qui découvre dans les œuvres du passé des significations que son époque même n'y avait pas aperçues; ce sont les univers originaux des écrivains de l'avenir qui éveillent dans les œuvres du passé d'étranges anticipations. Peut-être le phénomène se voit-il suggéré sous forme parodique à travers le sujet posé à Gisèle, pour la composition française du certificat d'études: «Sophocle écrit des Enfers à Racine pour le consoler de l'insuccès d'Athalie» ${ }^{43}$.

Instant dilaté de la lecture, temps d'une vie redisposé par la lecture, temps séculaire travaillant le sens des œuvres, temps disloqué révélant leurs plus grandes originalités: on ne saurait, chez Proust, séparer le processus de la lecture du phénomène ou de la catégorie du temps. Par quoi pour lui le fait de lire s'avère plus important que ce qui est lu. Qu'il lise le fictif Bergotte ou la marquise de Sévigné, le lecteur

(40) Correspondance, t. XIX, p. 643.

(41) Recherche, t. II, p. 838.

(42) Ibidem, p. 14.

(43) Ibidem, p. 264. 
installé dans sa durée vécue est le personnage central d'une philosophie de la lecture: qu'il la poursuive ou l'interrompe, s'y plonge ou s'en échappe, s'en représente les paysages ou en imagine les autres lecteurs, s'y installe en continuité ou y suscite des ruptures, il demeurera une conscience dans le temps, ne pouvant se constituer comme conscience de lecteur que dans le temps - Kant aurait dit, et Proust redit avec $\mathrm{Kant}^{44}$ : redisposant le monde de ses lectures, c'est-à-dire le monde, selon la forme $a$ priori de sa sensibilité au Temps.

LUC FRAISSE

Université de Strasbourg Institut universitaire de France

(44) Voir Proust et Kant, Actes du colloque de Strasbourg (19-21 novembre 2018) réunis par G. Bensussan et L. Fraisse, "Revue d'études proustiennes" 10, 2019/2. 